Article

\title{
Gender, Age and Subjective Well-Being: Towards Personalized Persuasive Health Interventions
}

\author{
Aisha Muhammad Abdullahi ${ }^{1, *(\mathbb{D})}$, Rita Orji ${ }^{2, *(\mathbb{D})}$ and Abdullahi Abubakar Kawu ${ }^{3}$ (D) \\ 1 Faculty of Computing, Federal University Dutse, Dutse P.M.B. 7156, Jigawa State, Nigeria \\ 2 Faculty of Computer Science, Dalhousie University, Halifax, NS B3H 4R2, Canada \\ 3 Ibrahim Badamasi Babangida University, Lapai 740005, Nigeria; abdullahikawu@ibbu.edu.ng \\ * Correspondence: am.abdullahi@fud.edu.ng (A.M.A.); rita.orji@dal.ca (R.O.)
}

Received: 16 August 2019; Accepted: 23 September 2019; Published: 27 September 2019

\begin{abstract}
Background: Subjective well-being (SWB) is an individual's judgment about their overall well-being. Research has shown that high subjective well-being contributes to overall health. SWB consists of both Affective and Cognitive dimensions. Existing studies on SWB are limited in two major ways: first, they focused mainly on the Affective dimension. Second, most existing studies are focused on individuals from the Western and Asian nations; (2) Methods: To resolve these weaknesses and contribute to research on personalizing persuasive health interventions to promote SWB, we conducted a large-scale study of 732 participants from Nigeria to investigate what factors affect their SWB using both the Affective and Cognitive dimensions and how distinct SWB components relates to different gender and age group. We employed the Structural Equation Model (SEM) and Confirmatory Factor Analysis (CFA) to develop models showing how gender and age relate to the distinct components of SWB; (3) Results: Our study reveals significant differences between gender and age groups. Males are more associated with social well-being and satisfaction with life components while females are more associated with emotional well-being. As regards age, younger adults (under 24) are more associated with social well-being and happiness while older adults (over 65) are more associated with psychological well-being, emotional well-being, and satisfaction with life. (4) Conclusions: The results could inform designers of the appropriate SWB components to target when personalizing persuasive health interventions to promote overall well-being for people belonging to various gender and age groups. We offer design guidelines for tailoring persuasive intervention to increase SWB based on an individual's age and gender group. Finally, we map SWB components to possible persuasive technology design strategies that can be employed to implement them in persuasive interventions design.
\end{abstract}

Keywords: subjective well-being; gender; age; persuasive health interventions; personalization

\section{Introduction}

Health and well-being are not just the absence of disease or illness, it is a combination of a person's social, mental, emotional and physical health [1]. Positive physical health and well-being may ultimately lead to other desirable human qualities such as increased vitality, productivity, longevity, and overall flourishing. Interventions to promote any or all of the components associated with health and wellbeing of individuals have gained some attention among researchers [2-4]. Subjective well-being (SWB) is an individual's judgment about their overall well-being and includes both Affective reactions (emotional, social and psychological well-being) and Cognitive judgments (satisfaction with life and happiness) [5,6]. Research has shown that there are relationships between physical health and the distinct components of SWB. For example, Kiecolt-Glaser et al. [7] showed that emotional distress can lead to slower wound healing in patients. It has also been found that psychological 
wellness promotes physical activity, healthy eating, and smoking cessation [8]. Therefore, health interventions that promote the individual components of the SWB will impact positively on people's overall physical health and well-being. There are several social strategies for persuasion (e.g., Reward, Competition, Social Comparison, Social Learning, Trustworthiness and Cooperation) and their effect in behavior change have been identified [9-14]. Given its value, PT has seen wide research application in domain such as health $[13,14]$ and education $[9,10]$. Persuasive technologies for health are designed as interventions with the primary purpose of changing a user's behavior or attitude without using coercion or deception [15]. PTs when appropriated in health applications can significantly impact on the outcomes [13,14], but it is even more impactful when it is personalized to user characteristics [12-14].

The idea of personalizing health interventions is being advocated for by Persuasive Technology (PT) researchers [15-18]. This is because individual differences have been shown to play an important role in peoples' SWB. For example, the influence of personality traits [19], income [20], gender and age [21], have been investigated by researchers and have been shown to influence people's SWB differently. However, most existing studies on the influence of individual differences on SWB focus on people from western and Asian countries, making their findings difficult to generalize to African countries. Also, existing literature focus only on one dimension of the subjective well-being, often, the Affective component, ignoring its other dimension, the Cognitive component. To bridge this gap, we investigate gender and age difference on the distinct components of SWB (emotional well-being, social well-being, psychological well-being, satisfaction with life and happiness), for people from Nigeria, to suggest ways that persuasive health interventions can be tailored to promote SWB of individuals from non-western cultures.

\section{Background}

In this section, we provide an overview of the distinct components of the subjective well-being and related works.

\subsection{Components of Subjective Well-Being}

SWB is a broad phenomenon that includes people's emotional responses, and global evaluation of life [5]. Diener et al. [22] reported SWB to consist of two distinct dimensions: (1) an Affective dimension (Hedonic) and (2) a Cognitive dimension (Eudemonic). In this section, we briefly discuss both dimensions:

\subsubsection{Affective (Hedonic) Dimension of SWB.}

The Affective dimension is a hedonic evaluation guided by emotions and feelings [22]. It reflects the amount of pleasant feelings and unpleasant feelings that people experience in their lives [22]. Peoples' psychological well-being, emotional well-being, and social well-being have been considered as Affective dimension of SWB [23].

1. Psychological well-being: Psychological well-being is an individuals' belief that there will be positive outcomes to events or circumstances [24] (p. 497). Research has shown that people who have positive psychological well-being are healthier generally [25]. Beliefs such as feeling secure about the future, being hopeful, being positive, being enthusiastic have been shown to promote people's psychological well-being $[24,26]$. Therefore, we hypothesize that a persuasive intervention designed to promote such believes in individuals will likely impact their psychological well-being and consequently their overall health and well-being. The psychological well-being scale developed by Ryff called Scales of Psychological Well-being (SPWB) has been used by some researchers to assess people's psychological well-being [26]. The scale is composed of six sub-scales in accordance with the six factors of positive functioning, namely: autonomy, environmental mastery, personal growth, purpose in life, positive relationship and self-acceptance [26]. However, the scale has been found to be statistically unreliable [27]. The Bradburn scale of psychological 
well-being is another scale that has been used to assess people's psychological well-being [26]. The scale is made up of two components: The Positive Affect component and the Negative Affect component. Each component is associated with 5 items used in assessing it, participant answers "Yes" or "No" to the items which ask whether they have had certain feelings in the past few weeks. The "No" score is subtracted from the "Yes" score to create a positive/negative affect difference score. Also, the psychological well-being scale developed by Diener et al. [28] has been widely used by researchers to assess people's psychological well-being. The psychological well-being scale developed by Diener et al. [28] consists of 12 items. Each is measured using a 5-point Likert scale, ranging from " $1=$ Strongly disagree" to " $5=$ Strongly agree". A response of 5 to an item indicates a high psychological well-being, and 1 indicates low psychological well-being. This study employs the psychological well-being scale developed by Diener et al. [28] which has been widely employed by researchers. A sample item includes: "I am competent and capable in the activities that are important to $\mathrm{me}^{\prime \prime}$.

2. Emotional well-being: Emotional well-being is defined as a feeling of relaxation and stress freeness [28]. It has been shown that emotionally healthy people have more stable mental health, thus, are healthier generally [29]. Activities that make people experience serenity, support, and company have been shown to promote people's sense of emotional well-being [23,28]. Hence, a persuasive intervention designed to promote such activities will likely impact people's emotional well-being and thus their overall health and well-being. Researchers have used the Warwick-Edinburgh Mental Well-being Scale (WEMWBS) developed by researchers at the Universities of Warwick and Edinburgh to assess people's emotional well-being [30]. WEMWBS is a 14-item scale, the scale is scored by summing responses to each item measured using a " 5 " point Likert scale. Also, the emotional well-being scale developed by Diener et al. [28] has been widely used by researchers to measure the emotional well-being of individuals. Therefore, in this study, we employ the emotional well-being scale developed by Diener et al. [28]. The scale consists of 16 items, each item in the scale is measured using a Likert scale ranging from " $1=$ Strongly disagree" to " $5=$ Strongly agree". A sample item includes: "I am competent and capable in the activities that are important to me".

3. Social well-being: Social well-being refers to an individual's interaction and relationship with others. [31]. Research has shown that the more social ties people have, the better their health [32]. For example, being married, having children, and social ties with formal organizations such as religious organizations can instill a sense of responsibility and concern for others that can lead one to engage in behaviors that protect the health of others as well as their own health. Thus, health interventions that provide opportunities for people to interact and work together with other people, share and compare the information with others, provide support and companionship to others may promote their social well-being and hence their overall health and wellbeing. Some researchers have used the Positive and Negative Affect Scale (PANAS) to assess the Affective dimension of Subjective well-being [28]. However, the social well-being scale developed by Huppert et al. [31] has been widely used by researchers to measure this component of SWB. Hence, we employ this scale to assess the social well-being of participants in this study. The scale consists of 16 items; each item is measured using a 5-point Likert scale ranging from " $1=$ Strongly disagree" to " $5=$ Strongly agree". A sample item includes: "I see myself as a part of my society".

\subsubsection{Cognitive (Eudemonic) Dimension of Subjective Well-Being}

The Cognitive dimension is an information-based appraisal of one's life for which people judge the extent to which their life so far measures up to their expectations and resembles their envisioned 'ideal' life [6]. People's satisfaction with life and happiness has been considered as a Cognitive dimension of SWB $[19,23]$.

1. Satisfaction with life: Satisfaction with Life is defined as people's personal judgment about their life. Specifically, it deals with people's belief that at least their life measures up favorably 
against their expectations or how a person feels about their life as a whole, over a long period of time $[33,34]$. Studies have shown that satisfaction with life influences health-related quality of life (HRQOL) [34]. For example, people who are satisfied with their life are less likely to involve themselves in excessive alcohol drinking, excessive eating or smoking. Meanwhile, these unhealthy behaviors can easily be adopted by people who feel their needs are not met to deal with the stress. Activities such as setting and achieving goals, attaining status, gaining respect, have been shown to promote people's Satisfaction with life [34,35]. Therefore, health interventions that provide opportunities for people to engage in such activities may promote their satisfaction with life and therefore their overall health and wellbeing. The Satisfaction with life scale developed by Diener et al. [33] has been widely used by researchers to measure this component of SWB. Therefore, in this study, we employ the Satisfaction with life scale developed by Diener et al. [33] to assess our participants 'satisfaction with life. The scale consists of 5 items; each item is measured using a 5 -point Likert scale ranging from " $1=$ Strongly disagree" to " $5=$ Strongly agree". A sample item includes: "So far, I have gotten the important things I want in life".

2. Happiness: Happiness is defined as the momentary feeling of intense joy or a positive state of emotion at a single point in time [36]. It has been shown that happy people are healthier [37]. Activities such as expressing gratitude, acts of kindness, savoring, optimism, committing to one's goals have been shown to promote people's feelings of happiness. A persuasive intervention designed to increase an individual's overall happiness may likely impact on their health and well-being. Recent years have witnessed the emergence of many happiness scales. The Oxford Happiness Questionnaire (OHQ) developed by Peter Hill [38] is a popular happiness scale used by researchers. The Oxford Happiness Questionnaire consists of 29 items measured on a 6-point Likert scale. Scores for this scale is calculated by summing the score for each item and dividing by 29 , final scores are then interpreted. For example, if a participant selects " 1 " for all the items on the scale, the final happiness score of the individual will be " 1 ". " 1 " is interpreted as "not happy". Another happiness scale is the Authentic Happiness Inventory (AHI) developed by Slain [39]. AHI consist of 24-item groups; each group contains five statements from which an individual chooses which statements best describe their feelings. The final happiness score is then interpreted on a scale of " 1 " to " 5 ", with 5 being the highest in terms of happiness. One of the most widely used happiness scale is the one developed by Lyubomirsky and Lepper [36]. The scale consists of 4 items, each item is measured using a 5-point Likert scale ranging from " $1=$ Very unhappy" to " 5 = Very happy". A sample item includes: "If you were to consider your life in general these days, how happy would you say you are". This study employs the Happiness scale developed by Lyubomirsky and Lepper [36]. See Appendix A for all the scales used in this study.

\subsection{Related Work}

The investigation into the relationship between gender, age, and SWB has received some attention among researchers. For example, Gutiérrez et al. [40] conducted a study of Spaniards to examine the relations between gender, age and different components of SWB. However, their study focused on a single dimension of SWB (the affective dimension) ignoring its other dimension (the cognitive dimension). Two components were used to assess the Affective dimension (Positive affect and Negative affect). Their study revealed that age (increase in the number of years) is associated with the Positive affect. As regards gender, females obtained lower scores than males in Negative affect.

Similarly, Ronald [41] conducted a study of participants from 65 non-African societies to investigate the relationship between gender, age, and SWB. The study used one component (Happiness) to assess participants' SWB. Ronald [41] revealed that females under 45 years are happier than males under 45 , while females above 45 are less happy than males above 45 .

A recent study by Agrawal et al. [21] also showed that there is a statistically significant relationship between gender, age and different components of SWB. In their study of Indians, they explored the relationship between some Socio-demographic variables and distinct SWB components. Although 
their study considered the two dimensions of SWB (Affective and Cognitive), they used only three components to measure SWB (Positive affect, Negative affect, and satisfaction with life). Their study revealed that older men (over 55 years) had a lower negative affect than younger men (under 36 years) while older women (over 55 years) had a significantly lower Negative affect than younger women (under 36 years). As regards satisfaction with Life, younger men (under 36 years) had lower scores than the older men (over 55 years).

Also, in a meta-study of European countries, Deaton [42] revealed that life satisfaction decreased with age, particularly in Eastern European and former Soviet Union countries. In another study, Shmotkin [43] used three components to assess SWB: Affective balance, Life satisfaction, and Self-Anchoring. They found that age correlated negatively with all the subscales of SWB. As regards gender, they found no significant gender difference in the SWB subscales.

Similarly, Bălţătescu and Giusta et al. [44,45] carried out studies of Romanians and people from the United Kingdom respectively. Using the three components to assess SWB: Satisfaction with life, Positive affect, and Negative affect, they found that, women have higher levels of Negative affect than men. As regards age, he found that life satisfaction declines with increasing age among this population.

This present study differs from existing studies in three major ways: One, we investigate a developing African nation (Nigeria) which is often overlooked by researchers. Two, we investigate the two dimensions of subjective well-being using five distinct components. This provides a richer insight into subjective well-being. Three, we offer design guidelines and design considerations for personalizing Persuasive health intervention aimed at promoting SWB targeted at various gender and age group.

\section{Materials and Methods}

This study was designed to investigate how to tailor persuasive health interventions to individuals, by examining how gender groups (Males and Females) and age group (Older adults and Younger adults) relate to the two SWB dimensions (Cognitive and Affective) using five components. The cognitive dimension was assessed using the Happiness and Satisfaction with life components while the Affective dimension was assessed using the Psychological well-being, Emotional well-being, and Social well-being components. We collected data from participants and used Structural Equational modeling (SEM) in AMOS 2.0 to investigate whether there is a difference between the gender groups and age groups with respect to their association with the distinct SWB components. The data collection was overseen by the Federal University of Dutse's research ethics committee. To guide this research, we formulated two main hypotheses:

Hypothesis 1 (H1). There is a difference between males and females in their association with the distinct components of SWB.

Hypothesis 2 (H2). There is a difference between older adults and younger adults in their association with the distinct components of SWB.

\subsection{Participants}

This study is based on data from 732 participants. The participants were drawn from several works of life in Nigeria: Traders, civil servants, paramilitary, university staff, and students. Participants willingly volunteered to participate in this study, so no incentives were provided to them. Paper-pencil questionnaires were handed out to participants in their workplaces. The questions took approximately $15 \mathrm{~min}$ to complete. No identifying information was collected from participants, except for their socio-demographic data which included gender and age. Overall, $52 \%$ of the 732 participants are males and $48 \%$ are females. With regards to age, $21 \%$ (16-24), 19\% (25-34), 17\% (35-44), 13\% (45-54), $13 \%(55-64), 11 \%(65-74)$, and $6 \%(75+)$. Since our study aims at investigating whether there are differences between the gender groups (males and females) and age groups (younger adults and older 
adults) in their association with the distinct SWB components, it is therefore important to state that for the age group analysis, we selected and considered participants belonging to two age groups that are clearly different which is the standard practice. Hence, we compared 16-24 (younger adults) and over 65 (older adults) to ensure that the ages are very distinct. As a result, the total sample involved in the age group analysis is 278, which is a good sample size for this type of analysis [46]. However, for the gender group analysis, the full sample of 732 was used. Table 1 is the descriptive statistic of our data by gender group and Table 2 is the descriptive statistic of our data by age group.

Table 1. Demographic of participants by gender group.

\begin{tabular}{cc}
\hline Criterion & $\mathbf{N}=732$ \\
\hline $\begin{array}{c}\text { Gender } \\
\text { Age }\end{array}$ & $21 \%(16-24), 19 \%(25-34), 17 \%(35-44), 13 \%(45-54), 13 \%(55-64), 11 \%(65-74)$, and $6 \%(75+)$ \\
\hline
\end{tabular}

Table 2. Demographic of participants by age group.

\begin{tabular}{cc}
\hline Criterion & $\mathbf{N}=\mathbf{2 7 8}$ \\
\hline Gender & Male $(59 \%)$, Female $(41 \%)$ \\
Age & $57 \%(16-24), 41 \%$ (over 65 years) \\
\hline
\end{tabular}

\subsection{Data Sampling}

Participants were recruited from Nigeria in 2018. Seven states were selected: Kano, Kaduna, Katsina, Kebbi, Sokoto, Jigawa, and Zamfara. 100 participants were randomly selected from all the states except for Kano were 132 participants were selected. Universities, colleges, government/private offices from these states were randomly enlisted and personally visited by the research team to recruit participants. After a short introduction of the study to the head of each organization, participants were then randomly selected and approached. The purpose of the study was explained to them and their consents were sort. A paper-pencil questionnaire was then given to each respondent. Most of the respondents completed the survey which took approximately $15 \mathrm{~min}$ to complete immediately. A small number of the respondents went home with the questionnaire booklet and returned them after a mutually agreed period (at most after $24 \mathrm{~h}$ ). Random sampling was used for convenience in the selection of organizations and respondents. However, in keeping with the research aim, the research team deliberately selected respondents from both genders and various age groups.

\subsection{Data Analysis}

In this study, we conducted Multi-Group Confirmatory Factor Analysis (MGCF) to investigate whether there are differences between the gender groups (males and females) and age groups (younger adults and older adults) with respect to their association with the distinct SWB components. As regards age groups, we selected and considered participants within 16-24 (younger adults) and over 65 (older adults) to ensure that the ages are very distinct.

\subsection{Validation of Study Instrument}

We conducted Confirmatory Factor Analysis (CFA) to test the validity of our study instruments. We established a correlation matrix between components of SWB (Latent variables), see Table 3. 
Table 3. Correlation matrix between components of SWB.

\begin{tabular}{cccccc}
\hline Components of SWB & PWB & EWB & SoWB & SWL & H \\
\hline PWB & 1 & & & & \\
EWB & 0.14 & 1 & & & \\
SoWB & 0.01 & 0.01 & 1 & & \\
SWL & 0.02 & 0.02 & 0.05 & 1 & \\
H & 0.06 & 0.04 & 0.03 & 0.12 & 1
\end{tabular}

Psychological well-being $=$ PWB, Emotional well-being $=$ EWB, social well-being $=$ SoWL, Satisfaction With life $=$ SWL, happiness $=\mathrm{H}$.

\section{Results}

We conducted Multi-Group Confirmatory Factor Analysis (MGCFA) to establish whether there are differences between gender groups (male and female) and between age groups (older adults and younger adults) with respect to their association with the individual components of the SWB. Our data satisfy all the assumptions necessary to conduct a Latent mean invariance test. Firstly, we tested for Configural invariance to confirm whether we have same structure across groups by running individual CFAs in gender groups and in age groups. The goodness-of-fit indices show that the hypothesized models were a good fit to the data. The respective goodness-of-fits indices gender group is as follows: the males' model: $(\chi 2 / \mathrm{df}=1.74$, RMSEA $=0.058, \mathrm{CFI}=0.931)$ and females' model: $(\chi 2 / \mathrm{df}=1.52$, RMSEA $=0.061, C F I=0.942)$. Regarding the age group, the goodness-of-fits indices is as follows: older adults' model: $(\chi 2 / \mathrm{df}=2.18, \mathrm{RMSEA}=0.063, \mathrm{CFI}=0.951)$ and younger adult model: $(\chi 2 / \mathrm{df}=2.26$, RMSEA $=0.072, C F I=0.938)$. The recommended value for CFI is $>0.90$, RMSEA recommended values is $<0.08$, the recommended value for $\chi 2 / \mathrm{df}$ is $<3$. Our data satisfies all the recommended goodness-of-fits indices.

Secondly, we performed Metric invariance test to investigate whether factor loadings on SWB components are equal across gender groups (male and female) and equal across age groups (older adult and younger adult). The models obtained were an acceptable fit, the chi-square difference between the configural invariance models and the metrically invariant model were not significant: Gender group $(\Delta \times 2=4.31, p>0.05)$, Age group $(\Delta \times 2=5.24, p>0.05)$. This shows that the SWB constructs have the same meaning across groups.

\subsection{Differences in the Latent Means Across Gender Groups}

We performed latent mean invariance tests to investigate whether males and females differ in the SWB constructs. The model $(\Delta \times 2=10, p>0.05)$ shows significant mean differences between males and females for emotional well-being, social well-being, and satisfaction with life. Specifically, emotional well-being is more associated with females $(\varphi=3.6, p<0.001)$ compared to males $(\varphi=1.3$, $p<0.001)$. Social well-being is more associated with males $(\varphi=4.2, p<0.001)$ compare to females $(\varphi=1.4, p<0.001)$. Satisfaction with life is highly associated with males $(\varphi=4.3, p<0.001)$ compared to females $(\varphi=1.2, p<0.001)$. The results for gender group is as summarized in Table 4 .

\subsection{Differences in the Latent Means Across Age Groups}

Similarly, we performed latent mean invariance tests to investigate whether older adults and younger adults differ in their relation with the SWB constructs. The model $(\Delta \times 2=11, p>0.05)$ show significant mean differences between older adults and young adults for all the SWB constructs. Specifically, psychological well-being is more associated with older adults $(\varphi=3.7, p<0.001)$ compare to younger adults $(\varphi=1.9, p<0.001$. Similarly, emotional well-being is more associated with older adults $(\varphi=4.5, p<0.001)$ compare to younger adults $(\varphi=1.1, p<0.001)$. Social well-being is more associated with younger adults $(\varphi=3.6, p<0.001)$ compare to older adults $(\varphi=1.7, p<0.001)$. Satisfaction with life is highly associated with older adults $(\varphi=3.9, p<0.001)$ compare to younger 
adults $(\varphi=1.7, p<0.001)$. Happiness is highly associated with younger adults $(\varphi=4.8, p<0.001)$ compared to older adults $(\varphi=1.8, p<0.001)$. The results for Age group is as summarized in Table 5 .

Table 4. Latent means for gender groups.

\begin{tabular}{ccccc}
\hline Components of SWB & $\begin{array}{c}\text { Latent Mean, } \boldsymbol{\varphi} \text { (phi) } \\
\text { Males }\end{array}$ & $\begin{array}{c}\text { Latent Mean, } \boldsymbol{\varphi} \text { (phi) } \\
\text { Females }\end{array}$ & $\boldsymbol{\Delta} \boldsymbol{p}$ & $\boldsymbol{p}$ \\
\hline Psychological well-being & 2.7 & 2.6 & 0.1 & $>0.5$ \\
Emotional well-being & 1.3 & 3.6 & 2.3 & $<0.001$ \\
Social well-being & 4.2 & 1.4 & 2.8 & $<0.001$ \\
Satisfaction with Life & 4.3 & 1.2 & 3.1 & $<0.001$ \\
Happiness & 3.2 & 3.2 & 0.0 & $>0.05$ \\
\hline
\end{tabular}

Table 5. Latent means for the age group.

\begin{tabular}{ccccc}
\hline Components of SWB & $\begin{array}{c}\text { Latent Mean, } \boldsymbol{\varphi}(\mathbf{p h i}) \\
\text { Older Adults }\end{array}$ & $\begin{array}{c}\text { Latent mean, } \boldsymbol{\varphi}(\mathbf{p h i}) \\
\text { Younger Adults }\end{array}$ & $\boldsymbol{\Delta} \boldsymbol{\varphi}$ & $\boldsymbol{p}$ \\
\hline Psychological well-being & 3.7 & 1.9 & 1.8 & $<0.001$ \\
Emotional well-being & 4.5 & 1.1 & 3.4 & $<0.001$ \\
Social well-being & 1.7 & 3.6 & 1.9 & $<0.001$ \\
Satisfaction with Life & 3.9 & 1.7 & 2.2 & $<0.001$ \\
Happiness & 1.8 & 4.8 & 3.0 & $<0.001$ \\
\hline
\end{tabular}

\section{Discussion}

This study presents the results from investigating whether different gender groups (males and females) and age groups (older adults and younger adults) respond differently to the distinct components of SWB. This study is unique in that such a relationship has not been empirically conducted in among African audience. In this section, we discuss the results in relation to various age and gender groups.

\subsection{Relationship between Gender and SWB Components}

Gender and Social Well-being: Our results show that males show a higher level of social well-being than their female counterparts. This means that males harbor higher levels of social well-being compared to females. This is probably because, in Nigeria, males are encouraged to spend time together so that they bond and build a sense of communal goal as they are regarded as protectors of their local communities and culture. For example, local communities in the northern part of Nigeria have several groups of males serving as vigilantes. These vigilante groups are formed to protect women and children within the communities. These men often attach so much value to building strong networks among themselves, as they regard it as a way of building a sense of communal goal. On the other hand, females tend to remain indoors (attending to house chores) and interact less with the outside community as enjoin by the Islamic faith, which is the predominant religion in the northern part of Nigeria. This finding implies that the overall health and well-being of females can be more promoted if activities that give them more sense of social well-being are enhanced. Hence, to promote the SWB of females in Nigeria the social aspect of their lives should be focused on. Therefore, we recommend that persuasive health interventions designers targeted at promoting well-being (hence overall health and well-being) of females in Nigeria should focus on designing to promote their social well-being.

There are several techniques that can be used in PT design to create opportunities for an individual to interact and relate with others (Social well-being). For example, persuasive techniques from the social support category of the Persuasive System Design (PSD) framework [47] such as the Social comparison, Cooperation, and Competition, which provide opportunities for people to share and compare information about their behavior, interact and work together with other people, and compete with others could be employed by designers to promote an individual's sense of social well-being. 
Gender and Satisfaction with Life: Our results show that males are highly associated with satisfaction with life components compared to females. This means that males are more satisfied with their life compared to females. This is not surprising as most non-western societies such as Nigeria are generally patriarchal societies. They afford males more privileges and right that makes life worth living such as an opportunity to engage in activities that elevate their status, explore life as it pleases them, earn respect, achieve their goals and aspirations. For example, in patriarchal societies, males tend to create environments for themselves where they are in control and are served by females. In most Nigerian families, males are reverenced, this, in turn, mirrors a sense of superiority and life satisfaction. According to Huebner et al. [48] personal control over one's daily life influences their satisfaction with life positively. On the other hand, females in such societies are constrained in their ability to take control of life events, which may, in turn, inhibit life fulfilling actions, thus, reflect a lower level of life satisfaction. Furthermore, Satisfaction with life to some extent has to do with how wealthy and influential people are $[15,37,41]$. Males are usually wealthier and more influential than females in patriarchal societies. Even when females are allowed to work, they generally earn less than males irrespective of whether they do the same job or not. Hence, females often depend on their male counterparts to survive. This offers a sense of security and stability to many Nigeria men [45]. This finding implies that the overall health and well-being of females can be enhanced if they are given opportunities to engage in activities that give them more sense of satisfaction with life. Hence, this finding suggests that to increase the subjective well-being of females, the activities that enhance their satisfaction with Life should be focused on. Thus, we recommend that persuasive health interventions designers aimed at promoting the overall subjective well-being among females in Nigeria should focus on designing to promote their satisfaction with Life.

There are persuasive techniques that can be used in PT design to promote satisfaction with life. For example, activities such as setting and achieving goals, attaining status, gaining respect, have been shown to promote people's satisfaction with life [35]. Consequently, persuasive strategies such as Goal setting which provides people with opportunities to set their goals and Feedback which evaluates people's performance and provides them with information about their progress and achievements could be employed to promote a sense of achievement and fulfillment for people. Similarly, the Recognition strategy which provides opportunities for people's achievements to be publicly recognized could be employed by designers to make people experience feelings of pride and satisfaction with life and hence improve their overall sense of well-being.

Gender and Emotional Well-being: Our results show that females are more associated with emotional well-being than their male counterparts. This means that females harbor higher levels of emotional well-being compared to males. In fact, emotional well-being emerged as the weakest contributor to the subject well-being of males. A plausible explanation for this is that the image of males as protectors and providers in the societies, creates a mindset in them from a very young age that makes them perceive being emotional as a sign of weakness and failure. In societies like Nigeria, males are less likely to discuss their emotions with others [49]. They are not believed or expected to experience anxiety, depression or any other form of emotional distress. As a result, they do not talk about their feelings or express emotions unless as an expression of anger. There is a stigma attached to any male who does not abide by this norm. Thus, they shy away from seeking the necessary support or help they need when they experience any form of emotional distress. On the other hand, females tend to discuss and get support, especially from one another, when they are emotionally distressed. This finding is supported by Goldshmidt et al. [50] who conducted a study of Syrians to investigate whether females are more verbally expressive of their emotions than males. They found that females used significantly more emotional words than males. This finding implies that the overall health and well-being of males can be enhanced if activities that give them more sense of emotional well-being are enhanced. Hence, this finding suggests that to increase the SWB of males, the activities that enhance their emotional well-being should be focused on. Therefore, we recommend that persuasive health 
interventions designers targeted at promoting well-being among males could focus on designing to promote their emotional well-being.

PT designers can employ some specific techniques to promote people's emotional well-being. For example, activities that make people experience serenity, love, support, company have been shown to promote people's sense of emotional well-being [28]. As a result, persuasive strategies such as Social facilitation, and Social learning could be implemented to provide opportunities for users to discern that other people are performing the behavior along with them and some social support, which gives them the will and boosts to continue the behavior change task, could be employed by designers to promote emotional well-being and hence overall health and well-being of individuals. Similarly, persuasive strategies such as Praise and Reward for any micro-achievement could be employed to encourage and show some love which in turn promotes emotional well-being.

\subsection{Relationship between Age and SWB Components}

Age and Psychological Well-being: Our results show that older adults (over 65 years) are more positively associated with the psychological well-being component compared to their younger counterparts (16-24 years). This means that older adults harbor higher levels of psychological well-being than younger adults. According to Ryff's model [26], a key element of psychological wellness is "purpose in life", which is a feeling that what we are doing with our lives has some meaning and purpose. This means that older people tend to engage themselves with activities that give them a sense of purpose and add meaning to their life than young people. A possible explanation for this finding according to Carstensen et al. [51] is that perceived limitations with respect to how long more they have to live tends to lead to a shift in mindset that makes older adults to focus more on activities they found meaningful. For example, after the age of 50, people tend to discover themselves more, hence they become more conscious of the limited time there is in life, more influential and therefore can afford to live a life of purpose and do only things that add meaning to their lives. On the other hand, younger people are mostly in the experimental stage, which is often full of struggles, life of uncertainty, and filled with many errors and failures. Therefore, younger adults have less appreciation for what they do, which can, in turn, lead to low psychological well-being. Another key element in Ryff's model of psychological well-being [26] is "Autonomy", which is the ability to resist social pressures and to make and pursue one's own decisions. This means that older people can better regulate their affairs, regardless of social influence, which can, in turn, reflect a high sense of Psychological well-being compared to their younger counterparts. This finding is supported by the study of Carstensen et al. [51] who found that older people have an increased ability to view their situations positively and that negative perceptions about life decline steadily with age. This finding implies that the overall health and well-being of younger adults can be more promoted if activities that give them more sense of psychological well-being is enhanced. Hence, this finding suggests that to increase the SWB of younger adults from Nigeria, activities that enhance their psychological well-being should be emphasized. Therefore, we recommend that when designing persuasive health interventions targeted at younger people, designers should emphasize activities that promote their psychological-being as a way of promoting their overall well-being.

In PT design, there are several techniques that can be used in promoting psychological well-being. For example, feeling secure about the future, being hopeful, being positive, being enthusiastic have been shown to promote people's psychological well-being [24,26]. Therefore, persuasive technology strategies such as Reward and Praise for small achievements have the power to evoke some feel-good emotions while Self-monitoring and Simulation that track and project the impact of an individual's micro efforts towards achieving the desired behavioral outcome can raise the anticipation of positive results hence promote psychological well-being.

Age and emotional Well-being: Similarly, our results show that older adults displayed a higher level of Emotional well-being than younger adults. This means that younger adults may be more emotionally unstable. A plausible explanation for this is that, with increasing age and experience, 
people seek out additional help and companionship from families and friends, as a result, an elderly person is much more likely to have more valuable relationship and companionship, which may, in turn, help them better manage their everyday affairs, including their emotions. Hence, they tend to be more emotionally stable. This is in line with the study of Taneva [52] who found that older people report better control of negative emotions than their younger counterparts. This implies that, the overall health and well-being of younger adults can be improved if activities that give them more sense of emotional well-being are enhanced. Therefore, we recommend that when designing persuasive health interventions targeted at younger adults, designers should also emphasize activities that promote their Emotional well-being as a way of promoting their overall well-being.

Age and Satisfaction with Life: Furthermore, our results show that older adults show a higher level of satisfaction with life than their younger counterparts. This means that older adults tend to be more satisfied with life than younger adults. According to Gutiérrez et al. [53] the most important factor that affects life satisfaction in older adults is the nature of the relationship with other people. One possible explanation for our finding is the influence of culture and religious beliefs. Participants in this study are from Nigerian where older adults live with their children and grandchildren, unlike in the western cultures where most older adults live in nursing homes and in isolation. This strong and valuable relationship with close family members, may, in turn, lead to a high sense of satisfaction with life for the elderly. Interestingly, this finding contradicts that of Deaton [42] and Bălţătescu [44] who found that life satisfaction decreased with age in Eastern Europeans. This is probably due to the role played by culture and how elderly people are treated in Western societies. However, Deaton [42] attributed the pattern to low health satisfaction and high levels of disability among the elderly in these countries. This implies that the overall health and well-being of younger adults from Nigeria can be improved if activities that give them more sense of satisfaction with life are enhanced. Therefore, we suggest that persuasive health interventions designers who aim to promote overall well-being among younger adults should emphasize activities that promote their satisfaction with life.

Age and Happiness: Our results show that younger adults are more associated with happiness. This means that younger adults tend to be happier than older adults in general. According to Lyubomirsky et al. [36] happiness is the momentary feeling of intense joy or a positive state of emotion at a single point in time. Happiness unlike satisfaction with life is short-lived [34]. One possible explanation why young adults are inclined to this kind of feeling is that young adults tend to often have parent-teen conflicts or experience academic pressure, as a result, may seek such short-lived excitement to help them ease off the stress after heated arguments with parents or get mental breaks after school pressure. This is probably the reason why young adults are fond of social media since the excitement that social media provides is usually short-lived [54]. On the other hand, older adults tend to drop or distance themselves from less intimate and short-lived relationships and investments more in relationships with long-lasting prospects. Thus, are not often exposed to experiencing a short-lived feeling of excitements (Happiness). This implies that the overall health and well-being of older adults can be more promoted if activities that give them more sense of Happiness are enhanced. Therefore, we recommend that when personalizing persuasive health interventions to older adults, designers could also focus on integrating activities that improve their feeling of happiness to promote their overall well-being.

There are techniques that can be used in PT design to promote Happiness. For example, activities and strategies such as expressing gratitude, acts of kindness, savoring, optimism, committing to one's goals have been shown to promote people's feeling of Happiness [55]. Therefore, persuasive strategies such as Rewards which give individuals credit for performing the target behavior and Praise, in recognition of good behaviors can be employed by persuasive intervention designers to promote health and well-being. Self-monitoring and Simulation can also be used to enable the user to see the projected and accumulated benefits of their tiny efforts towards achieving the desired behaviors as a way of promoting happiness and hence overall health and well-being. 
Age and Social Well-being: Also, our results show that younger adults are more associated with Social well-being than older adults. Given their developmental stage, having peer groups may be intriguing for most young adults. This is also coupled with the increasing number of social media whose target audience are mostly young adults. Hence, they are more likely to have larger social networks, get along more easily with people and interact more often than older adults. According to Faisal et al. [56] the low social interaction of older adults may be due to chronic illness and disability. This implies that the overall health and well-being of older adults can be promoted if activities that give them more sense of social well-being are enhanced. The importance and effects of social participation in the prevention and management of chronic diseases cannot be overemphasized. Therefore, we recommend that when designing persuasive health interventions targeted at older adults, designers should emphasize activities that promote their sense of social well-being to promote overall well-being.

In summary, females scored significantly lower in satisfaction with life and Social well-being components. Therefore, persuasive health interventions designers targeted at females should employ persuasive strategies that promote more, their sense of satisfaction with life and social well-being. However, when designing for males, designers should employ persuasive strategies that build more, their Emotional well-being which they scored least in. Similarly, younger adults scored significantly lower in Emotional well-being, followed by Satisfaction with life, and Psychological well-being. Therefore, designers aimed at improving the overall well-being of this population should employ persuasive strategies that emphasize these components: emotional well-being, followed by satisfaction with life, and psychological well-being. On the other hand, older adults scored significantly lower in Social well-being followed by Happiness. Therefore, designers should employ persuasive strategies that promote their sense of Social well-being and Happiness to improve their overall well-being. In general, emotional well-being and happiness emerged as the strongest predictor of subjective well-being for females while Satisfaction with life, followed by social well-being, and happiness are the strongest predictor of subjective well-being for males. With respect to age, happiness, followed by social well-being emerged as the strongest contributor to the subjective well-being of younger adults while emotional well-being, satisfaction with life, and psychological well-being are the strongest predictor of subjective well-being of older adults.

\section{Limitation}

One limitation of this study is that we used self-report measurements to assess people's SWB. Although this is still the standard practice, we acknowledge that it may be biased. Also, we used a random sampling technique for our data collection, while this is the common approach in this research area, we acknowledge that using a stratified random sampling technique for data collection would have made our findings more generalizable. Lastly, this study looked at the association between SWB components and two key factors (age and gender), our future work will look at a prospective cohort to investigate for possible causal relationship.

\section{Conclusions}

This paper presented the results of a large-scale study of 732 participants from a developing African country to investigate for gender and age differences with respect to what contributes to their Subjective Well-Being (SWB). We used five SWB components: emotional well-being, social well-being, psychological well-being, satisfaction with life and happiness. Our results show that males are more associated with social well-being and satisfaction with life while females are more associated with emotional well-being. As regards age groups, younger adults (under 24) are more associated with social well-being and happiness while older adults (over 65) are more associated with psychological well-being, emotional well-being, and satisfaction with life. Our findings can inform persuasive technology designers on how to tailor health interventions to people from developing African nations to increase their efficacy in promoting their subjective well-being based on their age 
and gender group. Our work offers four main contributions to the field of Persuasive Technology and health intervention design. First, we reinforce the need to personalize persuasive health interventions to targeting African audience by revealing that gender and age influence how Nigerians relate to distinct SWB components. Second, we establish that gender and age are important characteristic for personalizing persuasive health interventions targeting Nigerians. So far, none of the existing works investigated the relationship between gender and age and SWB among Nigerians. Third, we examine the relationship between individual gender and age and the different SWB components and develop design guidelines for personalizing persuasive health interventions to individuals based on their gender and age. Finally, we suggest some persuasive strategies to promote individual components of the SWB. This is an essential step toward developing personalized health applications that will effectively engage users and promote desired behavior change.

Author Contributions: A.M.A. led the research design, data collection and analysis, manuscript preparation, and editing. R.O. contributed to the research design, manuscript preparation, and review. A.A.K. participating in manuscript review and editing.

Funding: Rita Orji's research is funded by the NSERC Discovery Grant.

Acknowledgments: We would like to thank RandaTech for their assistance with analyzing the data and the study participants.

Conflicts of Interest: The authors declare no conflict of interest.

\section{Appendix A. Personality Traits and Subjective Well-Being Measurement Instrument}

Personality traits

On a scale of 1 to 5 ( $1=$ Strongly disagree to $5=$ Strongly agree $)$, to what extent do you agree with the following statements.

I see myself as someone who:

1. is reserved.

2. is generally trusting.

3. tends to be lazy.

4. is relaxed, handles stress well.

5. has few artistic interests.

6. is outgoing, sociable.

7. tends to find fault with others.

8. does a thorough job.

9. gets nervous easily.

10. has an active imagination.

Social well-being scale

On a scale of 1 to 5 ( $1=$ Strongly disagree, $5=$ Strongly agree $)$ to what extent do you agree with the following statement.

1. I have close contact with my direct neighbors.

2. I think it's important to be a member of an association.

3. I'm content with my social position.

4. I'm content with the relation to my neighbors.

5. People in my neighborhood handle each other in a positive manner.

6. I see myself as a part of society.

7. I gladly have contact with other people via social media (Facebook, e-mail).

8. There are enough people with who I feel strongly connected.

9. I gladly help other people if they need my help.

10. I'm content with the composition of the population in my neighborhood. 
11. I feel accepted in my neighborhood.

12. I trust in the people in my surrounding.

13. I gladly participate in activities in my neighborhood.

14. My work situation contributes to my well-being.

15. I gladly spent time with online gaming with other people.

16. I'm content with my surrounding.

Psychological well-being scale

On a scale of 1 to 5 ( 1 = Strongly disagree, $5=$ Strongly agree) to what extent do you agree with the following statement.

1. I lead a purposeful and meaningful life.

2. I am engaged and interested in my daily activities.

3. I am competent and capable in the activities that are important to me.

4. I am a good person and live a good life.

5. My material life (income, housing, etc.) is sufficient for my need

6. I am satisfied with my religious or spiritual life.

7. I am optimistic about the future.

8. I have no addictions, such as to alcohol, illicit drugs, or gambling

9. People respect me.

10. I have been feeling optimistic about the future.

11. I actively contribute to the happiness and well-being of others.

12. I generally trust others and feel part of my community.

Emotional well-being scale

On a scale of 1 to 5 ( $1=$ Strongly disagree, $5=$ Strongly agree) to what extent do you agree with the following statement.

1. I have been feeling useful.

2. I have been dealing with problems well.

3. I have been thinking clearly.

4. I have been feeling close to other people.

5. I have been feeling confident.

6. My social relationships are supportive and rewarding

7. I have been interested in new things.

8. I have not been feeling depressed.

9. I have not been feeling sad.

10. I have not been feeling afraid.

11. I have been feeling contented.

12. I have been feeling positive.

13. I have been feeling joyful.

14. I have been feeling cheerful.

15. I have been able to make up my own mind about things.

16. I have been feeling loved.

Happiness scale

On a scale of 1 to 5 ( $1=$ Very unhappy to $5=$ Very happy), please circle one number that corresponds to your response to each question.

1. These days, how happy or unhappy would you say you are?

2. Compared to most of your peers, you consider yourself? 
3. Some people are generally happy. They enjoy life regardless of what is going on, getting the most out of everything. To what extent does this characteristic describe you?

4. Some people are generally not happy, although they are not depressed, they never seem as happy as they might be. To what extent does this characteristic describe you?

5. Please, list things that make you happy (you can list up to 10).

6. please, list things that make you unhappy (you can list up to 10).

Satisfaction with life scale

On a scale of 1 to 5 ( $1=$ Strongly disagree, $5=$ Strongly agree $)$ to what extent do you agree with the following statements.

1. In most ways my life is close to my ideal.

2. The conditions of my life are excellent.

3. I am satisfied with my life.

4. So far, I have gotten the important things I want in life.

5. If I could live my life over, I would change almost nothing.

6. All things considered; I am satisfied with my life these days.

7. Please, list things that gives you satisfaction in life (you can list up to 10 things).

8. Please, list things that makes you unsatisfied with life (you can list up to 10 things).

\section{References}

1. Fuhrer, M.J. Subjective Well-Being: Implications for Medical Rehabilitation Outcomes and Models of Disablement. Am. J. Phys. Med. Rehabil. 1994, 73, 358-364. [CrossRef] [PubMed]

2. Thieme, A.; Wallace, J.; Meyer, T.D.; Olivier, P. Designing for mental wellbeing. In Proceedings of the 2015 British HCI Conference, Lincoln, UK, 13-17 July 2015; pp. 1-10.

3. Brey, P.B. Design for the Value of Human Well-Being. In Handbook of Ethics, Values, and Technological Design: Sources, Theory, Values and Application Domains; Springer: Berlin, Germany, 2015; pp. 365-382.

4. Desmet, P.M.; Pohlmeyer, A.E.; Forlizzi, J. Special issue editorial: Design for subjective well-being. Int. J. Des. 2013, 7, 1-3.

5. Dolan, S.; Robert, M. Measuring subjective wellbeing: Recommendation on measures for use by national governments. J. Soc. Pol. 2012, 2, 409-427. [CrossRef]

6. Van Hoorn, A.; Castriota, S. A short introduction to subjective well-being: Its measurement, correlates and policy uses. In Proceedings of the International Conference: Is Happiness Measurable and What Do Those Measures Mean for Policy, Rome, Italy, 2-3 April 2007; pp. 2-7.

7. Kiecolt-Glaser, J.K.; Loving, T.J.; Stowell, J.R.; Malarkey, W.B.; Lemeshow, S.; Dickinson, S.L.; Glaser, R. Hostile marital interactions, proinflammatory cytokine production, and wound healing. Arch. Gen. Psychiatry 2015, 62, 1377-1384. [CrossRef] [PubMed]

8. Department of Health. Our Health and Wellbeing Today. 2010; No. 3; p. 68. Available online: https://assets.publi shing.service.gov.uk/government/uploads/system/uploads/attachment_data/file/215911/dh_122238.pdf (accessed on 12 September 2019).

9. Orji, F.A.; Oyibo, K.; Orji, R.; Greer, J.; Vassileva, J. Personalization of Persuasive Technology in Higher Education. In Proceedings of the 27th ACM Conference on User Modeling, Adaptation and Personalization, Larnaca, Cyprus, 9-12 June 2019.

10. Abdullahi, A.M.; Orji, R.; Nwokeji, J.C. Personalizing Persuasive Educational Technologies to Learners' Cognitive Ability. In Proceedings of the 2018 IEEE Frontiers in Education Conference (FIE), San Jose, CA, USA, 3-6 October 2018; pp. 1-9.

11. Abdullahi, A.M.; Oyibo, K.; Orji, R. The influence of cognitive ability on the susceptibility to persuasive strategies. In Proceedings of the Personalization in Persuasive Technology Workshop, Persuasive Technology 2018, Waterloo, ON, Canada, 17 April 2018; pp. 22-33.

12. Abdullahi, A.M.; Orji, R.; Oyibo, K. Personalizing Persuasive Technologies: Do Gender and Age Affect Susceptibility to Persuasive Strategies? In Proceedings of the 26th Conference on User Modeling, Adaptation and Personalization, Singapore, 8-11 July 2018; pp. 329-334. 
13. Ndulue, C.; Rita, O. STD PONG: A Personalized Persuasive Game for Risky Sexual Behavior Change in Africa. In Proceedings of the Personalization in Persuasive Technology Workshop, Persuasive Technology 2018, Waterloo, ON, Canada, 17 April 2018.

14. Orji, R.; Oyibo, K.; Lomotey, R.K.; Orji, F.A. Socially driven persuasive health intervention design: Competition, social comparison, and cooperation. Health Inform. J. 2018. [CrossRef] [PubMed]

15. Orji, R.; Mandryk, R.L. Gender, Age, and Responsiveness to Cialdini's Persuasion Strategies. In Proceedings of the International Conference on Persuasive Technology, Chicago, IL, USA, 3-5 June 2015; pp. 147-159.

16. Orji, R.O.; Vassileva, J.; Mandryk, R.L. Modeling gender differences in healthy eating determinants for persuasive intervention design. In Proceedings of the International Conference on Persuasive Technology, Sydney, Australia, 3-5 April 2013; pp. 161-173.

17. Orji, R. Exploring the persuasiveness of behavior change support strategies and possible gender differences. In Proceedings of the Second International Workshop on Behavior Change Support Systems, Padua, Italy, 22 May 2014; Volume 1153, pp. 41-57.

18. Orji, R.; Mandryk, R.L.; Vassileva, J. Gender and persuasive technology: Examining the persuasiveness of persuasive strategies by gender groups. In Proceedings of the 9th International Conference on Persuasive Technology (PERSUASIVE 2014), Padova, Italy, 21-23 May 2014; pp. 48-52.

19. Ha, S.E.; Kim, S. Personality and Subjective Well-Being: Evidence from South Korea. Soc. Indic. Res. 2013, 111, 341-359. [CrossRef]

20. Diener, E.; Biswas-Diener, R. Will Money Increase Subjective Well-Being? Soc. Indic. Res. 2002, 57, 119-169. [CrossRef]

21. Agrawal, J.; Murthy, P.; Philip, M.; Mehrotra, S.; Thennarasu, K.; John, J.P.; Girish, N.; Thippeswamy, V.; Isaac, M. Socio-demographic Correlates of Subjective Well-being in Urban India. Soc. Indic. Res. 2011, 101, 419-434. [CrossRef]

22. Diener, E.; Oishi, S.; Lucas, R.E. Personality, Culture, and Subjective Well-Being: Emotional and Cognitive Evaluations of Life. Annu. Rev. Psychol. 2003, 54, 403-425. [CrossRef]

23. Chamberlain, K. The Structure of Subjective Well-Being. Soc. Indic. Res. 1988, 20, 581-604. [CrossRef]

24. Compton, W.C. Towards a tripartite factor structure of mental health: Subjective well-being, personal growth and religiosity. J. Psychol. 2001, 135, 486-500. [CrossRef] [PubMed]

25. Harris, M.; Magy, M.; Don, M. The Relationship between Psychological Well-Being and Perceived Wellness in Graduate-Level Counseling Students. High. Learn. Res. Commun. 2006, 34, 14-31.

26. Van Dierendonck, D.; Díaz, D.; Rodríguez-Carvajal, R.; Blanco, A.; Moreno-Jiménez, B. Ryff's six-factor model of psychological well-being, a Spanish exploration. Soc. Indic. Res. 2008, 87, 473-479. [CrossRef]

27. The Ryff Scales of Psychological Well-Being. 2005. Available online: https://centerofinquiry.org/uncategorize d/ryff-scales-of-psychological-well-being (accessed on 12 September 2019).

28. Diener, E.; Robert, B.-D. Happiness: Unlocking the Mysteries of Psychological Wealth; Wiley/Blackwell: New York, NY, USA, 2008.

29. Positive Emotions and Well-Being. Available online: www.psychologytoday.com/us/blog/between-cultures /201611/positive-emotions-and-wellbeing (accessed on 24 October 2018).

30. Stewart-Brown, S.; Mohammed, K.J. Warwick-Edinburgh Mental Well-Being Scale (WEMWBS): development and UK validation. Annu. Rev. Psychol. 2001, 52, 141-166.

31. Huppert, F.A.; Marks, N.; Clark, A.; Siegrist, J.; Stutzer, A.; Vittersø, J.; Wahrendorf, M. Measuring Well-being across Europe: Description of the ESS Well-being Module and Preliminary Findings. Soc. Indic. Res. 2009, 91, 301-315. [CrossRef]

32. Umberson, D.; Montez, J.K. Social Relationship and Health: A flashpoint for health policy. J. Health Soc. Behav. 2010, 51, 54-66. [CrossRef]

33. Diener, E.D.; Emmons, R.A.; Larsen, R.J.; Griffin, S. The Satisfaction with Life Scale. J. Pers. Assess. 1985, 49, 71-75. [CrossRef]

34. Prasoon, R.; Chaturvedi, K.R. Life satisfaction: A literature review. Res. J. Manag. Humanit. Soc. Sci. 2016, 1, 1-32.

35. Strine, T.W.; Chapman, D.P.; Balluz, L.S.; Moriarty, D.G.; Mokdad, A.H. The associations between life satisfaction and health-related quality of life, chronic illness, and health behaviors among U.S. community-dwelling adults. J. Community Health 2008, 33, 40-50. [CrossRef] 
36. Lyubomirsky, S.; Lepper, H.S. A measure of subjective happiness: Preliminary reliability and construct validation. Soc. Indic. Res. 1999, 46, 137-155. [CrossRef]

37. Lyubomirsky, S.; King, L.; Diener, E. The Benefits of Frequent Positive Affect: Does Happiness Lead to Success? Psychol. Bull. 2005, 131, 803-855. [CrossRef] [PubMed]

38. Argyle, M.; Hills, P. The Oxford Happiness Questionnaire (OHQ): A compact scale for the measurement of psychological well-being. Personal. Individ. Differ. 2002, 33, 1073-1082.

39. Şanlı, E.; Çelik, S.B.; Gençoğlu, C. Validity and Reliability of the Authentic Happiness Scale', Khazar. J. Hum. Soc. Sci. 2019, 22, 5-20. [CrossRef]

40. Gutiérrez, J.L.G.; Jiménez, B.M.; Hernández, E.G.; Pcn, C. Personality and subjective well-being: Big five correlates and demographic variables. Personal. Individ. Differ. 2005, 38, 1561-1569. [CrossRef]

41. Ronald, I. Gender, Aging, and Subjective Well-Being. Int. J. Comp. Sociol. 2002, 43, 391-408.

42. Deaton, A. Income, aging, health and wellbeing around the world: Evidence from the Gallup World Poll. J. Econ. Behav. Organ. 2007, 22, 53-72.

43. Shmotkin, D. Subjective well-being as a function of age and gender: A multivariate look for differentiated trends. Soc. Indic. Res. 1990, 23, 201-230. [CrossRef]

44. Bălţătescu, S. Gender and Age Differences in Subjective Well-being: Romania 1990-2005. In Gender, Lifespan and Quality of Life; Springer: Dordrecht, The Netherlands, 2014; pp. 99-114.

45. Giusta, M.D.; Jewell, S.L.; Kambhampati, U.S. Gender and Life Satisfaction in the UK. Fem. Econ. 2011, 17, 1-34. [CrossRef]

46. Koh, K.H.; Zumbo, B.D. Multi-Group Confirmatory Factor Analysis for Testing Measurement Invariance in Mixed Item Format Data. J. Mod. Appl. Stat. Methods 2008, 7, 471-477. [CrossRef]

47. Oinas-kukkonen, H.; Harjumaa, M. Persuasive Systems design: Key Issues Process Model and System Features. Commun. Assoc. inf. Syst. 2009, 24, 28. [CrossRef]

48. Huebner, E.S.; Suldo, S.M.; Smith, L.C.; McKnight, C.G. Life satisfaction in children and youth: Empirical foundations and implications for school psychologists. Psychol. Sch. 2014, 41, 81-93. [CrossRef]

49. Denrele, A. Men Do Suffer from Emotional Abuse. Vanguard News 2015, 12, 5-6.

50. Goldschmidt, O.T.; Weller, L. Talking Emotions: Gender Difference in a Variety of conventional Context. Symb. Interact. 2000, 23, 117-138. [CrossRef]

51. Carstensen, L.L.; Fung, H.H.; Charles, S.T. Socioemotional Selectivity Theory and the Regulation of Emotion in the Second Half of Life. Motiv. Emot. 2016, 27, 103-123. [CrossRef]

52. Taneva, S. What is psychological well-being and how it changes throughout the employment cycle? In Work, Health and Sustainability: Building Citizenship; UnicampBFCM: Campinas, Brazil, 2016; pp. 83-90.

53. Gutiérrez, M.; Tomás, J.M.; Galiana, L.; Sancho, P.; Cebrià, M.A. Predicting life satisfaction of the Angolan elderly: A structural model. Aging Ment. Health 2013, 17, 94-101. [CrossRef]

54. Social Media and Happiness: The Unfortunate Truth. 2015. Available online: www.kulraj.org (accessed on 2 February 2019).

55. Life Potentials: Types of Happiness in Psychology. 2014. Available online: www.theworldcounts.com (accessed on 2 February 2019).

56. Faisal, H.G.; Sutan, R.; Elnajeh, M.; Abdalqader, M.A.; Baobaid, M. The importance of social participation and networking among elderly people: Short review. J. Manag. Sci. 2017, 15, 99-107.

(C) 2019 by the authors. Licensee MDPI, Basel, Switzerland. This article is an open access article distributed under the terms and conditions of the Creative Commons Attribution (CC BY) license (http://creativecommons.org/licenses/by/4.0/). 